\title{
Multi-height structures in injection molded polymer
}

\section{Andersen, Nis Korsgaard; Taboryski, Rafael J.}

\section{Published in:}

Microelectronic Engineering

Link to article, DOI:

10.1016/j.mee.2015.03.017

Publication date:

2015

Document Version

Peer reviewed version

Link back to DTU Orbit

Citation (APA):

Andersen, N. K., \& Taboryski, R. J. (2015). Multi-height structures in injection molded polymer. Microelectronic Engineering, 141, 211-214. https://doi.org/10.1016/j.mee.2015.03.017

\section{General rights}

Copyright and moral rights for the publications made accessible in the public portal are retained by the authors and/or other copyright owners and it is a condition of accessing publications that users recognise and abide by the legal requirements associated with these rights.

- Users may download and print one copy of any publication from the public portal for the purpose of private study or research.

- You may not further distribute the material or use it for any profit-making activity or commercial gain

- You may freely distribute the URL identifying the publication in the public portal

If you believe that this document breaches copyright please contact us providing details, and we will remove access to the work immediately and investigate your claim 


\title{
Multi-height structures in injection molded polymer
}

\author{
Nis K. Andersen ${ }^{\dagger}$ and Rafael Taboryski ${ }^{\dagger}$ \\ ${ }^{\dagger}$ Department of Micro- and Nanotechnology, Technical University of Denmark, 2800 Kongens Lyngby, Denmark
}

\section{Abstract}

We present the fabrication process for injection molded multi-height surface structures for studies of wetting behavior. We adapt the design of super hydrophobic structures to the fabrication constrictions imposed by industrial injection molding. This is important since many super hydrophobic surfaces are challenging to realize by injection molding due to overhanging structures and very high aspect ratios. In the fabrication process, we introduce several unconventional steps for producing the desired shapes, using a completely random mask pattern, exploiting the diffusion limited growth rates of different geometries, and electroforming a nickel mold from a polymer foil. The injection-molded samples are characterized by contact angle hysteresis obtained by the tilting method. We find that the receding contact angle depends on the surface coverage of the random surface structure, while the advancing contact angle is practically independent of the structure. Moreover, we argue that the increase in contact angle hysteresis correlates with the concentration of pinning sites among the random surface structures.

\section{Introduction}

Since the discovery of the special surface structures giving rise to super hydrophobic surfaces in nature researchers have sought to artificially replicate the effect by micro and nano structuring surfaces. It is widely known that the most common cause of super hydrophobicity is due to trapping of air between surface structures when liquid is applied on the surface. This composite state, the socalled Cassie-Baxter state [1], facilities low adhesion and high apparent contact angles of liquid drops on the surface. The Cassie Baxter state is often energetically unfavorable and can collapse into the wetting state, the so called Wenzel state [2], by various transitions $[3,4]$. In the Wenzel state, liquid drops adhere to the surface. Keeping the droplets in the Cassie-Baxter state is therefore of key importance for maintaining super hydrophobicity [5]. Here we present a fabrication technique for realizing multi-height structures for super-hydrophobic purposes. By introducing multi-height structures, the Cassie Baxter state cannot collapse directly into the Wenzel state but will encounter several mixed states during the transition, see Figure 1. 


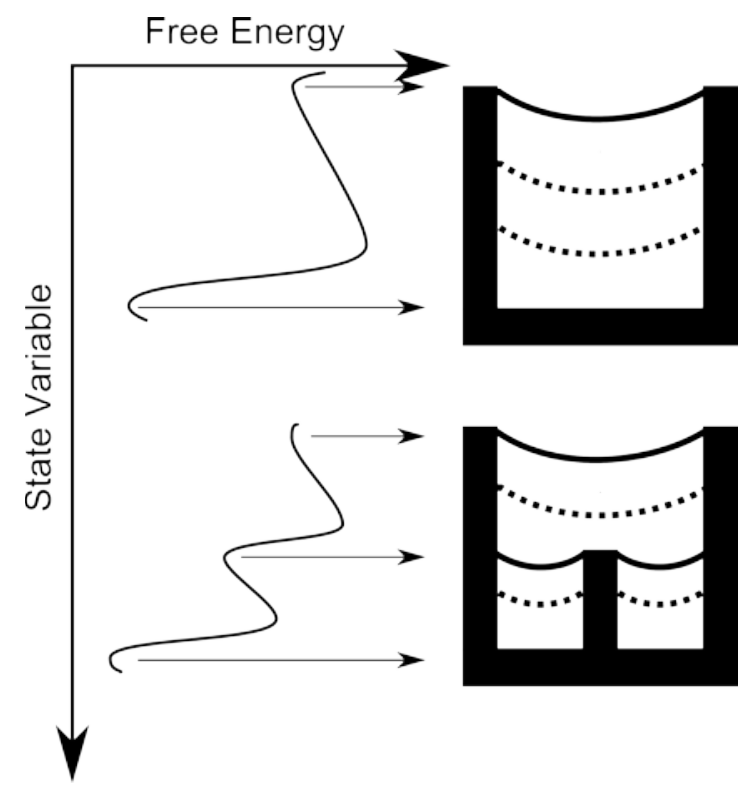

Figure 1. Sketch illustrating the energy associated with the state of the system, solid lines being stable or meta-stable states and dashed lines being wetting transitions. By introducing a second structure height, an extra meta-stable state appears in the energy landscape.

For all mixed states, the adhesion between the drop and the surface is between the extreme values of the Cassie Baxter state and the Wenzel state. This creates a gradual change in adhesion of the drop in contrast to the binary behavior seen for single height structures [5].Overall, the multiple structure heights increases the number of states where the drop is able to roll off, thereby increasing the robustness of the super hydrophobicity. Many different approaches have been shown to achieve a super hydrophobic surface, including lithographic patterning [6], self-assembling structures [7], maskless processes [8] and direct replication of natural water repellant surfaces [9]. In this respect, we focus on structuring the super hydrophobic surface by injection molding. Many of the artificial and natural super hydrophobic surfaces seen in literature are undesirable for injection molding since they rely on high aspect ratios or overhanging structures [10]. Therefore, we present a fabrication process comprising overlapping random patterns that gives rise to multilevel surface structures without overhang. These surface structures are designed to improve the stability of the Cassie Baxter state, reduce the resistance for drops rolling off the surface, and are realized by industrial injection molding. For this we choose multi-height, low aspect ratio, microstructures with rounded corners. The low aspect ratio is a constraint imposed by the production method and would normally result in an unstable Cassie Baxter state. The stability is sought improved by introducing multiple heights in the surface structures. Finally, as proposed by Krumpfer et al. [11], we developed a method to round the corners of the microstructures for reduction of roll-off resistance. 


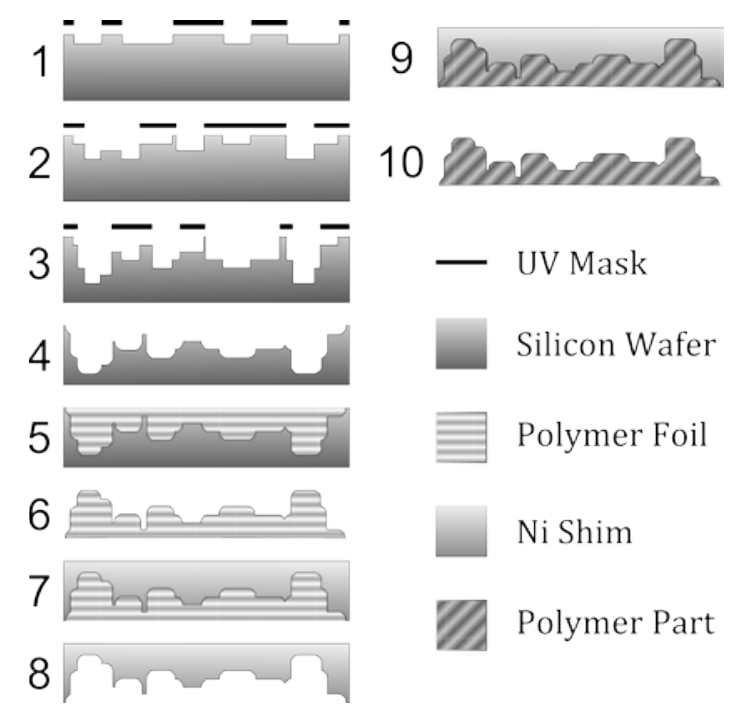

Figure 2. Schematic drawing of the fabrication process for the multi-height structures in injectionmolded polymer. 1-3) UV Lithography and D-RIE etching, 4) Thermal oxide growth and removal in BHF, 5-6) Imprint in polymer foil, 7-8) Electroforming of Ni, 9-10) Injection molding of final part.

The fabrication of the final injection molded polymer sample can be broken down to a chain of steps shown in Figure 2. The fabrication steps can also be divided into 4 different phases; fabrication of the master structures in a silicon wafer (1-4), reversing the polarity using hot embossing (5-6), creating mold insert by electroforming (7-8) and injection molding with polypropylene (9-10). In order to get the desired rounding of protrusions we grow and remove a thermal oxide on the silicon surface. During oxidation the growth rate of the oxide is diffusion limited, resulting in different rounding at open and closed corners. This is shown in Figure 3, where a circular pillar is oxidized and shown in profile. The corners at the tops of the pillars remain sharp while the corners at the base of the pillars are smoothened out. To utilize this effect to produce protrusions with rounded tops we define the structures as holes in the silicon master, oxidize it and reverse the polarity by imprinting in a polymer foil. 


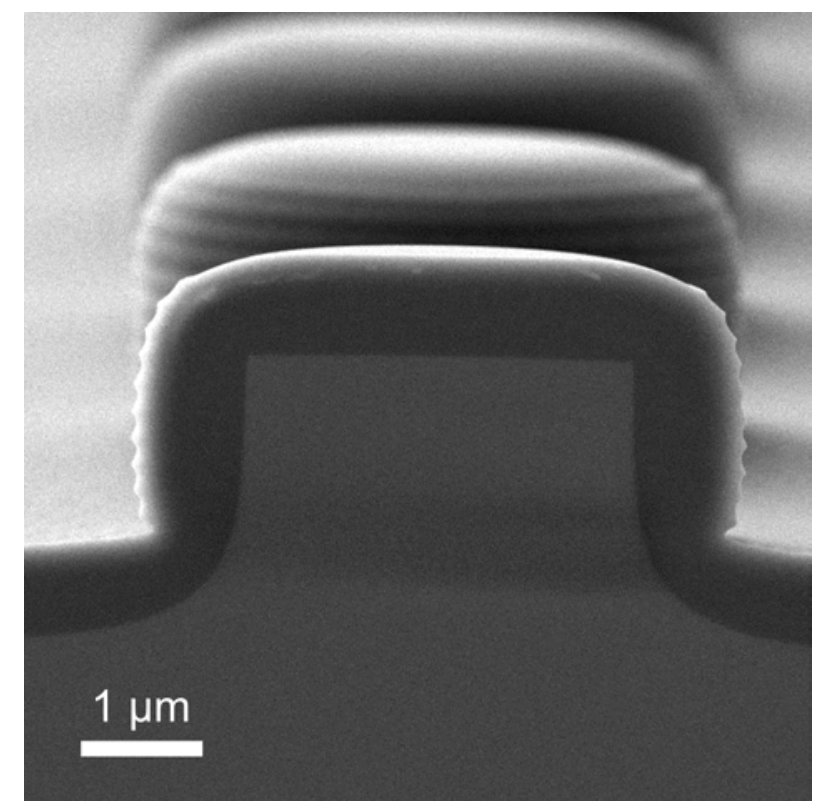

Figure 3. SEM image of a cleaved Si pillar covered with thermally grown oxide. The morphological difference between oxide grown at open (top of pillar) and closed (base of pillar) corners is clearly visible.

The mask used to define the surface structures consists of a matrix with $16(4 \times 4)$ fields of $6 \times 6 \mathrm{~mm}^{2}$ areas where surface coverage is varied along one axis $(0.22 \%, 0.26 \%, 0.30 \%, 0.33 \%)$ and the diameter of the dots is varied along the other $(5 \mu \mathrm{m}, 7 \mu \mathrm{m}, 9 \mu \mathrm{m}$ and $11 \mu \mathrm{m})$. To predict the specific surface coverages we simulate the structuring. The surface coverage is calculated as a function of the number of dots $\mathrm{k}$ by $f=1-\left(1-\pi r^{2} / A\right)^{k}$, where $\mathrm{r}$ is dot radius, and $\mathrm{A}$ is area of the pattern. This equation is derived from the probability of a point in $A$ being covered by at least one of $k$ circles with radius $r$. The probabilistic nature of this equation makes it a very good approximation for a macroscopic area covered by microscopic dots. The local microscopic surface coverage might however deviate significantly from the macroscopic value.

\section{Experimental}




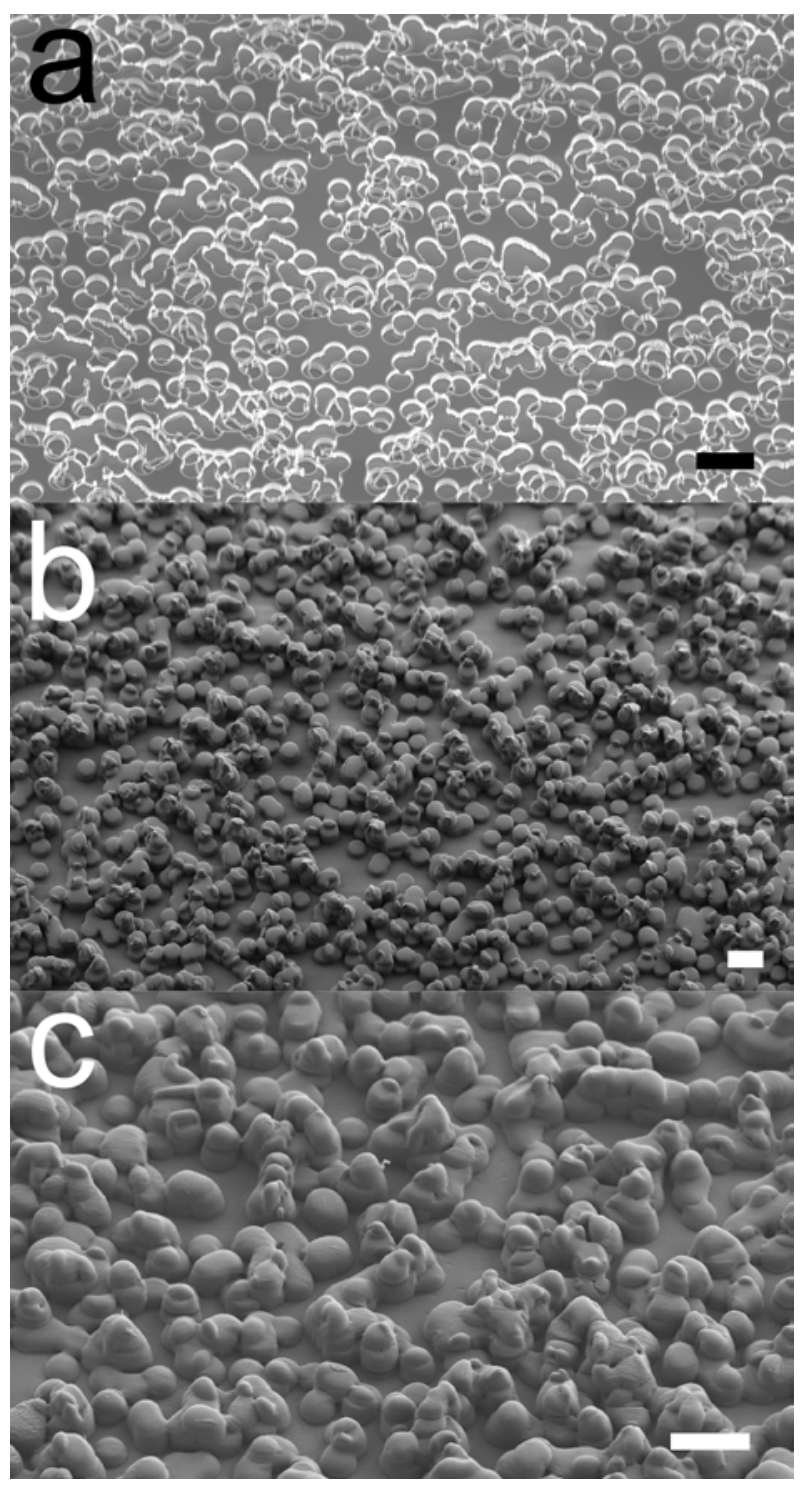

Figure 4. SEM image of surface structures during the fabrication process. a) Structures in silicon after three consecutive lithography and etching steps. b) Reversed structures in polymer foil after imprint. c) Final structure in injection molded polypropylene. Scale bar is $20 \mu \mathrm{m}$ and all samples are tilted $30^{\circ}$.

To fabricate the silicon master for the Nickel (Ni) insert we used a single crystalline silicon (Si) wafer. Etching of microstructures was a three-step process that was repeated three consecutive times for creating the multi-height structures. First step is spin coating the silicon wafer with $4.2 \mu \mathrm{m}$ AZ5214E photoresist and soft baking at $90^{\circ} \mathrm{C}$ for 60 s (Maximus 804 , ATMsse Gmbh). Second step is UV exposure by $57 \mathrm{~mJ} / \mathrm{cm}^{2}$ (EVG620, EVG) and development for 60s (AZ 351B developer mixed 1:5 with water). Third step is pattern transfer by D-RIE etching (Pegasus DRIE, STS). The remainder of the photoresist was removed by Oxygen plasma (Plasma processor 300, Tepla). The random multi-height surface structure is achieved by etching to three different depths in each repetition and misaligning the 
mask by $0.5 \mathrm{~mm}$ between each exposure. Each misalignment is done perpendicular to the previous step resulting in $5 \times 5 \mathrm{~mm}^{2}$ fields with the desired structure. The etched structure is shown in Figure $4 a$. For the wafer presented in this paper we used etching depths of $2,8 \mu \mathrm{m}, 3,8 \mu \mathrm{m}$ and $6,6 \mu \mathrm{m}$ resulting in structure heights of $0 \mu \mathrm{m}, 2,8 \mu \mathrm{m}, 3,8 \mu \mathrm{m}, 6,6 \mu \mathrm{m}, 9,4 \mu \mathrm{m}, 10,4 \mu \mathrm{m}$, and 13,2 $\mu \mathrm{m}$ depending on the combination of random overlaps of holes. By etching the smallest amount $(2,8 \mu \mathrm{m})$ during first etching and largest amount $(6,6 \mu \mathrm{m})$ during last etching the problems with spinning photoresist into deep trenches is minimized. If one tries to etch higher aspect ratio holes, the spinning of photoresist often fails. This can easily be seen by the naked eye and the photoresist can be removed and reapplied before etching, only resulting in a longer process time. To smoothen out structures, $1 \mu \mathrm{m}$ of wet thermal oxide was grown and consecutively removed in BHF buffer. The silicon wafer was then coated with FDTS by molecular vapor deposition (MVD100E, Applied Microstructures inc.). Polarity of the structures was reversed by imprinting (CNI, Nil Technology) the structures into a $300 \mu \mathrm{m}$ thick polymer foil (TOPAS $8007 \mathrm{~s} 04$ ) at $160^{\circ} \mathrm{C}$ under 6 bar for $20 \mathrm{~min}$. The foil was peeled off the silicon master on a hotplate at $78^{\circ} \mathrm{C}$. Structures imprinted in the foil are shown in Figure $4 \mathrm{~b}$. The foil was then sputter coated with $100 \mathrm{~nm}$ NiV (Custom system, Kurt J. Lesker). With then NiV thin film acting as a seed layer the polymer foil was electroplated (Microform 200, Technotrans) with Ni to a thickness of 300 $\mu \mathrm{m}$. To prepare the nickel shim for use in the injection molder the polymer foil is dissolved in Toluene at room temperature overnight, this releases the foil and the insert is then cleaned in Toluene at $80^{\circ} \mathrm{C}$ for 1 hour two times. Before using the insert in the injection molder, the insert is coated with an FDTS anti-stiction layer (MVD100E, Applied Microstructures inc.). The nickel shim was then mounted in an industrial injection molder (Victory 80/45 Tech, Engel) used to produce the final polypropylene (HD120MO, Borealis) samples using a variothermal molding process. During the molding process, the mold is heated to $120^{\circ} \mathrm{C}$ before injection and then cooled to $50^{\circ} \mathrm{C}$ before release. The injection molded surface structures are shown in Figure 4c. The microstructures on the produced parts were characterized by confocal microscopy (PLu Neox 3D Optical Profiler, Sensofar) using a 50x objective with a numerical aperture of 0.95 .

To characterize the wetting behavior of the injection molded polymer samples we have measured the advancing and receding contact angles by the tilting plate method. Prior to every measurement, any surface charge remaining from the molding process was neutralized using ionized air (Zerostat 3, Milty). For all measurements we used $10 \mu \mathrm{L}$ drops, tilting the sample at $1 \%$ s capturing images of the drop profile with 1 frame per second (Attension Theta optical tensiometer). For each measurement, we obtain a sequence of images. During the tilting, we often see the drop in a stick slip motion while sliding down the sample surface. We measure the advancing $\left(\theta_{A}\right)$ and receding $\left(\theta_{R}\right)$ contact angles on the last frame before the drop completely leaves the surface. Contact angles have been determined using both polynomial and manual fitting. The two different methods provide results with much smaller deviation than the scatter between different measurements.

\section{Results and discussion}

To verify the multi-scale properties of the surface structures on the produced part we measured the topography on all spaces with confocal microscopy. A histogram of the height data for the space containing $5 \mu \mathrm{m}$ features with 33\% coverage on the photo-mask is shown in Figure 5 . On the histogram each etching step is clearly seen as a peak ( $A, B$ and $C)$, with the extra heights resulting 
from overlaps of the random structures $(A+B, A+C, B+C$ and $A+B+C)$. The resulting microstructure therefore have 7 different height levels after 3 etching steps. We have analyzed the height distribution for all 16 fields and all show multi-height properties. As one would expect the height distribution for structures produced with lower surface coverage on the photo-mask, have smaller peaks. Likewise, the structures with larger feature size have sharper peaks due to less contribution from rounded edges.

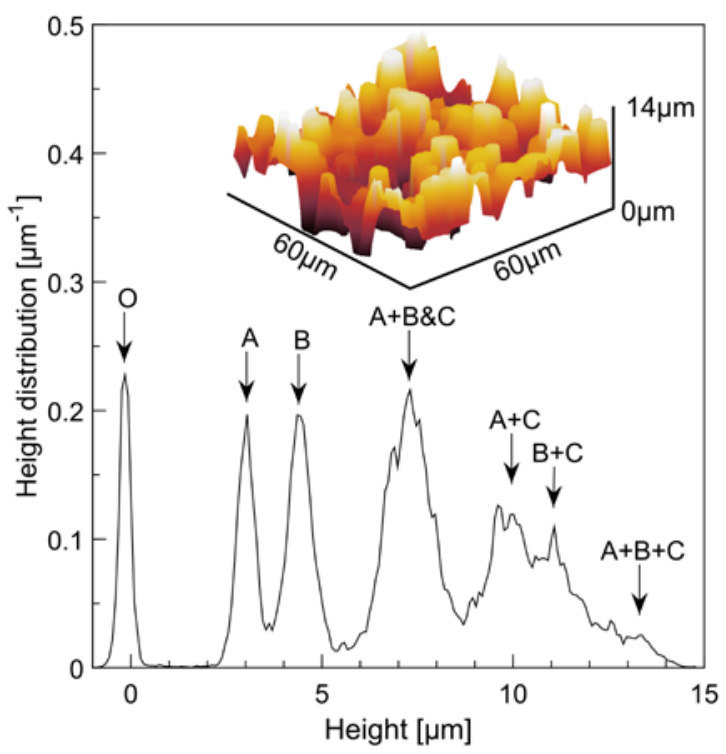

Figure 5. Height distribution obtained by confocal microscopy of the field with $5 \mu \mathrm{m}$ pillars and a surface coverage of $33 \%$. In the height distribution, the multi-scale feature is clearly seen from the three consecutive etching steps A, B and C. With the random overlaps of etched areas, also the sum of the etching depths appears as peaks in the histogram. The insert shows a 3D reconstruction of the surface structure.

Dynamic contact angles were measured on the 12 different geometries on five different samples and the collected results are plotted in Figure 6 . We did not measure any dynamic contact angles for the surface structures with a diameter of $11 \mu \mathrm{m}$ since the drops were stuck even at $90^{\circ}$ tilt for these structures. The contact angle hysteresis should in theory scale with the contact area between solid and liquid [12] but due to the random multi-height structures this value is difficult to determine. Instead, we plot the hysteresis as a function of the surface coverage on the mask used to produce the surface. For all surfaces the advancing angle is around $160^{\circ}$ and the difference in hysteresis originates only from the receding angle. This is in agreement with previous findings for drops in the Cassie Baxter state rolling off the surface $[13,14]$.. To optimize the geometry for increased water repellency one normally decreases the surface coverage of structures in contact with the water at the cost of decreasing the stability of the Cassie Baxter state [15]. For the random multi-height structures, we see that this in addition seems to lead to an increased hysteresis. This observation we ascribe to the local variation in the concentration of protrusions. The random nature of the surface structures will result in a normal distribution of local surface coverage around the macroscopic average value. In local regions with low microscopic surface coverages, the Cassie Baxter state will collapse resulting in a local pinning of the drop in those regions [16]. The decrease of macroscopic surface coverage will thereby 
increase the number of pinning sites, resulting in higher contact angle hysteresis. The stick-slip behavior seen during the tilting experiment supports this explanation. . This can further be understood in the following way: The drop rests in an area with a given contact angle hysteresis; at a certain tilting angle the drop starts moving down the slope; if the drop encounters an area with higher contact angle hysteresis the current tilting angle is not enough to keep the drop rolling and it stops. This phenomenon repeats several times during the measurement until the tilting angle is high enough to detach the drop from the area with the highest degree of pinning.

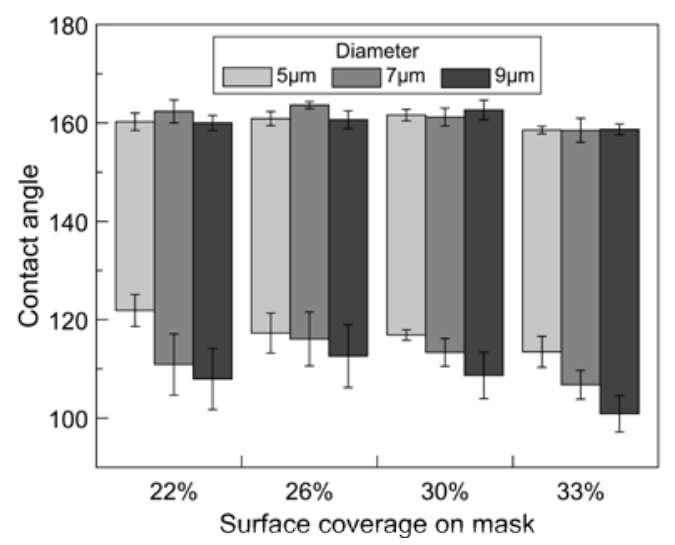

Figure 6. Contact angle hysteresis data for the different surface geometries, where the bottom and top of each bar is the measured receding and advancing contact angle, respectively. The hysteresis on the $11 \mu \mathrm{m}$ surface could not be measured due to complete pinning of the drop. For the lowest surface coverage structures, the hysteresis increases due to an increased number of pinning sites in the random surface. Error bars are standard deviation for measurements on five samples.

\section{Conclusion}

We have shown the fabrication process for the production of random multi-height structures in injection molded polypropylene. This we do using a randomly patterned mask, repeated etching and lithography steps to create random overlaps between features. To get the desired topography we utilize the asymmetrical growth of oxide in open and closed corners combined with hot embossing to get the desired polarity. To characterize the wettability we measured the contact angle hysteresis on the produced surfaces using the tilting plate method. We see the expected trend of decreasing contact angle hysteresis with decreasing surface coverage for most of the surfaces but for the lowest surface coverage, the hysteresis increases. This we ascribe to the increase in pinning sites on random surfaces with low surface coverage. An increase in the number of random pinning sites will stand out on tilting experiments since a drop rolling down the surface will tend to stick to the area with the highest local contact angle hysteresis.

\section{Acknowledgements}

This work is supported by the Danish National Advanced Technology Foundation (HTF) through the "Advanced Technology Platform NanoPlast" (grant no. 007-2010-2). 


\section{References}

[1] A.B.D. Cassie, S. Baxter, Wettability of porous surfaces, Trans. Faraday Soc. 40 (1944) 546. doi:10.1039/tf9444000546.

[2] R.N. Wenzel, RESISTANCE OF SOLID SURFACES TO WETTING BY WATER, Ind. Eng. Chem. 28 (1936) 988-994. doi:10.1021/ie50320a024.

[3] E. Søgaard, N.K. Andersen, K. Smistrup, S.T. Larsen, L. Sun, R. Taboryski, Study of Transitions between Wetting States on Microcavity Arrays by Optical Transmission Microscopy., Langmuir. (2014). doi:10.1021/la502855g.

[4] E. Bormashenko, Progress in understanding wetting transitions on rough surfaces., Adv. Colloid Interface Sci. (2014) 1-12. doi:10.1016/j.cis.2014.02.009.

[5] A. Lafuma, D. Quéré, Superhydrophobic states., Nat. Mater. 2 (2003) 457-60. doi:10.1038/nmat924.

[6] R. Fürstner, W. Barthlott, C. Neinhuis, P. Walzel, Wetting and self-cleaning properties of artificial superhydrophobic surfaces., Langmuir. 21 (2005) 956-61. doi:10.1021/la0401011.

[7] L. Gao, T.J. McCarthy, A perfectly hydrophobic surface (thetaA/thetaR $=180$ degrees $/ 180$ degrees)., J. Am. Chem. Soc. 128 (2006) 9052-3. doi:10.1021/ja062943n.

[8] G. Sun, T. Gao, X. Zhao, H. Zhang, Fabrication of micro/nano dual-scale structures by improved deep reactive ion etching, J. Micromechanics Microengineering. 20 (2010) 075028. doi:10.1088/0960-1317/20/7/075028.

[9] S.E. Lee, K.W. Lee, J.-H. Kim, K.-C. Lee, S.S. Lee, S.U. Hong, Mass-producible superhydrophobic surfaces., Chem. Commun. (Camb). 47 (2011) 12005-7. doi:10.1039/c1cc14489h.

[10] E. Celia, T. Darmanin, E. Taffin de Givenchy, S. Amigoni, F. Guittard, Recent advances in designing superhydrophobic surfaces., J. Colloid Interface Sci. 402 (2013) 1-18. doi:10.1016/j.jcis.2013.03.041.

[11] J.W. Krumpfer, P. Bian, P. Zheng, L. Gao, T.J. McCarthy, Contact angle hysteresis on superhydrophobic surfaces: an ionic liquid probe fluid offers mechanistic insight., Langmuir. 27 (2011) 2166-9. doi:10.1021/la105068c.

[12] W. Choi, A. Tuteja, J.M. Mabry, R.E. Cohen, G.H. McKinley, A modified Cassie-Baxter relationship to explain contact angle hysteresis and anisotropy on non-wetting textured surfaces., J. Colloid Interface Sci. 339 (2009) 208-16. doi:10.1016/j.jcis.2009.07.027. 
[13] S.T. Larsen, N.K. Andersen, E. Søgaard, R. Taboryski, Structure irregularity impedes drop rolloff at superhydrophobic surfaces., Langmuir. 30 (2014) 5041-5. doi:10.1021/la5007633.

[14] A.T. Paxson, K.K. Varanasi, Self-similarity of contact line depinning from textured surfaces., Nat. Commun. 4 (2013) 1492. doi:10.1038/ncomms2482.

[15] D. Hee Kwon, S. Joon Lee, Impact and wetting behaviors of impinging microdroplets on superhydrophobic textured surfaces, Appl. Phys. Lett. 100 (2012) 171601. doi:10.1063/1.4705296.

[16] C.W. Extrand, Criteria for Ultralyophobic Surfaces, Langmuir. 20 (2004) 5013-5018. doi:10.1021/la036481s. 